\title{
A New Twofold Bargaining Game Paradigm for Wireless Energy Harvesting Algorithm
}

\author{
Sungwook Kim \\ Department of Computer Science, Sogang University, 35 Baekbeom-ro (Sinsu-dong) Mapo-gu, Seoul 04107, Republic of Korea \\ Correspondence should be addressed to Sungwook Kim; swkim01@sogang.ac.kr
}

Received 11 May 2021; Revised 21 June 2021; Accepted 22 July 2021; Published 30 July 2021

Academic Editor: Ding Xu

Copyright ( $\odot 2021$ Sungwook Kim. This is an open access article distributed under the Creative Commons Attribution License, which permits unrestricted use, distribution, and reproduction in any medium, provided the original work is properly cited.

Considering the characteristics of radio-frequency-based energy harvesting technology, a number of smart devices are assisted by energy-constrained relays, which need to harvest energy from the hybrid access point (HAP). To design a novel energy harvesting mechanism, we investigate the integration of simultaneous wireless information and power transfer (SWIPT) and wireless powered communication network (WPCN) for dual-hop wireless communications. In our proposed scheme, we design a twofold bargaining game paradigm to adaptively make control decisions for conflicting requirements. According to two different bargaining solutions, we reveal the conflicting relationship of energy harvesting and information transferring and develop two time sharing procedures. In a compact iterative manner, these procedures work together to maximize the dual-hop communication system performance. Through simulation results, we conduct a numerical analysis and prove that our twofold bargaining approach leads to a higher system performance compared with the existing state-of-the-art protocols.

\section{Introduction}

Internet of Things (IoT) is a key application paradigm for the future generation wireless communication systems. With the advent of IoT concept, novel applications, such as smart mirrors, robot vacuum cleaners, and wireless kitchen appliances, have been proposed, and Internet-interactive smart devices would transform our lives. In the upcoming era of the $6 \mathrm{G}$ communication technology, smart homes, pollution control, energy saving, smart transportation, and smart industries have changed the traditional way of living into a high-tech life style. Today, there are millions of smart IoT devices with an annual growth rate of around $25 \%$, and the number is expected to reach 80 billion by 2030 . The smart IoT paradigm will radically change the method we think about Internet and technologies in the $6 \mathrm{G}$ communication networks [1-3].

Recent advancements in IoT technologies have appealed to researchers and developers worldwide. However, there are still a lot of challenges and issues that need to be addressed to achieve the full potential of IoT impacts. Particularly, some wireless devices are powered by batteries that have limited operational time; therefore, the lifetime of these devices depends on their battery life. Although the lifetime of energy-constrained devices can be extended by replacing or recharging the batteries, it may be inconvenient, costly, dangerous in toxic environment, or even impossible. These challenging issues have boosted the development of an alternative approach. Recently, wireless energy transfer technology has drawn wide attention and significant interests since it potentially provides unlimited power supplies to the energy-constrained devices. To prolong the device's lifetime, this technology can make smart devices harvest the energy from wireless signals for their intended applications $[3,4]$.

As one category of existing energy transfer techniques, the radio frequency- (RF-) enabled technology provides the feasibility of a long-range energy transfer compared to other technologies, such as inductive coupling and magnetic resonance coupling technologies. Particularly, the RF signal is able to carry information and energy at the same time; this feature gives rise to RF-enabled wireless powered communications. Furthermore, recent advance in designing highly efficient rectifying antennas will enable more efficient 
wireless energy harvesting from RF signals in the near future. Therefore, the RF-enabled energy harvesting technique promises tremendous scope for the replacement of small batteries in low power electrical devices and systems $[4,5]$.

The research in RF energy harvesting mainly falls into two broad categories: simultaneous wireless information and power transfer (SWIPT) and wireless powered communication network (WPCN). The idea behind SWIPT is that IoT devices are able to split the received signal into two parts: one part for data communications and the other part for energy harvesting. From the same received signal, IoT devices transfer the information data while harvesting their energy. The concept of WPCN is that energy-limited IoT devices first harvest energy radiated from the signals transmitted by RF energy sources and then utilize this harvested energy for their communication needs. Therefore, IoT devices in the WPCN exploit the harvested RF energy to power their data transmissions according to a typical harvest-then-transmit protocol. Recently, both SWIPT and WPCN have been combined to enhance the efficiency of RF energy harvesting technologies [6-8].

Despite extensive efforts to develop the wireless energy techniques, most of the existing researches did not concern the cooperative control approach in a coordinated manner. In this paper, we consider the joint platform of SWIPT and WPCN ( $S$ - $W$ joint platform) to optimize the point-to-point wireless communication and energy harvesting. By modifying the conventional harvest-then-transmit protocol, the uplink and downlink control mechanisms for wireless communications are differently implemented. Specifically, the WPCN is used in the downlink and the SWIPT is employed in the uplink. In this way, we should formulate the time adjustment problem with respect to diverse demands. To solve this problem, it is necessary to design an effective control algorithm while adaptively mediating conflicting issues. However, this work is complex and challenging. Therefore, we need a new control paradigm.

1.1. Technical Concepts. Cooperative bargaining game is a process in which players decide how to share a surplus that they can jointly generate [9]. The reference aspiration bargaining situation (RABS) is one of the first studies which incorporated the notion of a reference point into the simple bargaining model. The RABS is formally defined to be the maximal feasible point on the line joining the aspiration point and disagreement point where the aspiration point is derived from the reference point instead of the disagreement point. Therefore, the RABS selects the individual maximum payoff at which each player's payoff gain from his disagreement point to aspiration point while maintaining the same proportion to the payoff. In the RABS, disagreement point has a role to play as a threat in the bargain while measuring the proportional payoffs [10].

The negotiated aspiration bargaining solution (NABS) is conceptually related to the proportional bargaining solutions, which choose the best feasible point along the line starting at disagreement point and proceeding up in a direction determined exclusively by an exogenous vector of bargaining weights. Particularly, the NABS proposes the best allocation in the direction of utopia starting at an endogenous reference point which depends on both the utopia point and bargaining power. A characterization for the NABS is obtained by relaxing Pareto optimality while adding the standard axiom of restricted contraction independence, plus the minimal condition that utopia should be selected if available $[11,12]$.

1.2. Main Contributions. According to the RABS and NABS, we can effectively handle the energy harvesting and dual-hop wireless communications in the $S-W$ joint platform. By considering the mutual interaction of multiple devices, we develop a new twofold bargaining game model to adjust the uplink's time duration for each service. At the first bargaining process, the time duration for each IoT device in the uplink is decided based on the concept of RABS. At the second bargaining process, the ratio of each relay's energy harvesting and uplink data communications is dynamically adjusted according to the idea of NABS. In our twofold bargaining model, control decisions are made to reach a mutually acceptable agreement among multiple IoT devices. Based on the step-by-step interactive and repeated fashion, our proposed approach leads to an appropriate performance balance in the dual-hop communications while effectively harvesting energy to maximize the system throughput.

1.3. Related Work. Recently, there has been an upsurge of research interests in RF energy harvesting technique, which is the capability of converting the received RF signals into electricity. The paper [13] provides a game-based fair resource allocation algorithm based on the cognitive radio network. By adopting the Stackelberg and supermodular game models, a cooperation protocol is developed to schedule the behaviors of primary network and secondary users. In [14], a distributed power splitting algorithm is designed to derive a profile of power splitting ratios. By using noncooperative games, each link is modeled as a strategic player who aims to maximize its own achievable rate. The paper [15] focuses on the energy efficiency based robust optimization with imperfect channel state information and nonlinear energy harvesting models in the SWIPT aided network. In particular, this study adopts a realistic robust beamforming design by employing the physical layer security technology and the SWIPT technology based on a nonlinear energy harvesting model. To deal with it, the fractional programming problem is transformed into a subtractive one by employing Dinkelbach's method. Then, successive convex approximation and the semidefinite relaxation method are used to obtain a treatable form [15].

In [6], Ramezani and Jamalipour proposed the Dual-hop System Throughput Maximization (DSTM) scheme for the WPCN system. The major goal of the DSTM scheme is to maximize the total system throughput [6]. The Relayassisted Dual-hop Throughput Maximization (RDTM) scheme considers a dual-hop wireless power transfer network consisting of a HAP, multiple users, and multiple energy-constrained relays [7]. In the RDTM scheme, the 
achievable downlink and uplink throughput maximization problem is formulated with respect to time allocation and power splitting coefficients for the dual-hop wireless communication platform [7]. The Wireless Communication Throughput Maximization (WCTM) scheme is developed for the dual-hop WPCN infrastructure with a full-duplex HAP, which can transmit energy to the energy-constrained relays and receive information from the IoT devices assisted by the relays simultaneously [8]. In the WCTM scheme, the amplify-and-forward mode and the decode-and-forward mode are considered, and the solution of throughput optimization problem is obtained through a low-complexity algorithm [8].

\section{The Proposed Wireless Powered Communication Scheme}

\subsection{RF-Enabled Energy Transfer and Twofold Bargaining} Model. Traditionally, one major observation has been made in wireless communications, which is that the far-off devices have to transmit with more power than the near devices. It results from the severe signal attenuation experienced by further devices from the hybrid access point (HAP). This unfairness problem is called the doubly near-far problem, which is caused by the difference of the distances from the IoT devices to the HAP. To mitigate this problem, energyconstrained relays are considered, where these relays can charge their batteries by harvesting the energy through the received RF signal from the HAP and then assist wireless communications between the HAP and their corresponding devices in an amplify-and-forward manner by consuming the harvested energy. With the harvest-then-cooperate concept, this new method is defined as a dual-hop wireless communication mechanism. To improve the efficiency of this mechanism, the $S-W$ joint platform is developed; it combines the WPCN in the downlink and the SWIPT in the uplink to harvest energy at relays through both signals coming from the HAP and IoT devices [7].

In this study, we consider a dual-hop wireless communication platform consisting of one HAP, $n$ IoT devices $\mathbb{N}=\left\{\mathscr{D}_{1}, \ldots, \mathscr{D}_{n}\right\}$, and $n$ energy-constrained relays $\mathbb{R}=\left\{\mathscr{R}_{1}, \ldots, \mathscr{R}_{n}\right\}$, where each agent is equipped with a single antenna to transfer energy and information. We assume that there are no direct links between the HAP and IoT devices, and relays are responsible for assisting the data transmission of IoT devices and the HAP. To be specific, $\mathscr{R}_{i}$ is exclusively used for forwarding the data between $\mathscr{D}_{i}$ and the HAP. The HAP and IoT devices are with stable energy supply, but energy-constrained relays need to harvest the energy from RF signals broadcast by the HAP and IoT devices. That is to say, relays harvest their energy through the downlink signal from the HAP and uplink signal from its corresponding IoT device, and then retransmit the device's data to the HAP by consuming the harvested energy. The overview of dual-hop wireless communication platform is shown in Figure 1 [6-8].

To maximize the performance of dual-hop wireless communication system, we propose a new RF energy harvesting scheme based on the $S$ - $W$ joint platform. In the proposed scheme, the control issues of downlink and uplink operations are related to the time sharing strategy. At time $t_{c}$, the allocated time duration in the WPCN downlink $\left(\tau_{0}\right)$ is used only for the energy transfer; the HAP broadcasts RF energy signal and the relays harvest their energy through the received signal. On the other hand, the time duration of SWIPT uplink $\left(L_{t}^{\mathrm{UP}}\right)$ is shared by $n$ relays, and the time duration $\tau_{i}$ is allocated to the $\mathscr{R}_{i}$ where $L_{t}^{\mathrm{UP}}=\sum_{1 \leq i \leq n} \tau_{i} . \tau_{i}$ is split by two time subslots, i.e., $\left[\left(1-\rho_{i}\right) \cdot \tau_{i}\right]$ and $\left[\rho_{i} \cdot \tau_{i}\right]$; $\left[\left(1-\rho_{i}\right) \cdot \tau_{i}\right]$ is used to transfer the data and energy from $\mathscr{D}_{1 \leq i \leq n}$ to $\mathscr{R}_{1 \leq i \leq n}$, and $\left[\rho_{i} \cdot \tau_{i}\right]$ is used to retransfer the data from $\mathscr{R}_{1 \leq i \leq n}$ to the HAP. To effectively adapt the current dual-hop communication environments, the $\rho_{i}$ value should be dynamically adjusted to maximize the system throughput. $\mathfrak{M}$ is the total bandwidth amount in the $S-W$ joint platform. The downlink and uplink frame structure in the $S$ - $W$ joint platform is illustrated in Figure 2 [6-8].

At each time $t$, we formulate the time sharing problem as a twofold bargaining game $(\mathbb{G})$ where $\mathbb{G}$ consists of the first $\left(\mathbb{G}^{F}\right)$ and second $\left(\mathbb{G}_{1 \leq i \leq n}^{S}\right)$ bargaining games. In $\mathbb{G}^{F}$, IoT devices in the $\mathbb{N}$ are game players, and the uplink time duration $\left(L_{t}^{\mathrm{UP}}\right)$ is shared through the concept of RABS. In $\mathbb{G}_{i}^{S}$, $\mathscr{R}_{i}$ in $\mathbb{R}$ adjusts its own $\rho_{i}$ value based on the idea of NABS. For the second bargaining process, multiple $\mathbb{G}_{1 \leq i \leq n}^{S}$ games are operated in a parallel manner. Therefore, $\mathbb{G}^{F}$ and $\mathbb{G}_{i}^{S}$ are repeated sequentially during the step-by-step iteration, and the time sharing process is operated interactively at each time step $t_{c} \in\left\{t_{1}, \ldots, t_{c}, t_{c+1}, \ldots\right\}$. Formally, we define the $\mathbb{G}$ game entities, i.e., $\mathbb{G}=\left\{\mathbb{G}^{F}, \mathbb{G}_{1 \leq i \leq n}^{S}\right\}=\left\{\mathbb{N}, \mathbb{R}, \mathfrak{M},\left\{\mathbb{G}^{F} \mid \mathbb{N}, \tau_{1 \leq i \leq}\right.\right.$ $\left.\left.n, U_{\mathscr{D}_{1 \leq i \leq n}}\right\},\left\{\mathbb{G}_{1 \leq i \leq n}^{S} \mid \mathbb{R}, \rho_{1 \leq i \leq n}, \mathcal{U}_{\mathscr{R}_{1 \leq i \leq n}}\right\}, T\right\}$ of gameplay:

(i) In $\mathbb{G}, \mathbb{G}^{F}$ and $\mathbb{G}_{1 \leq i \leq n}^{S}$ are mutual and reciprocal interdependent in an interactive manner, and they work together to share each limited time step.

(ii) $\mathbb{N}$ is the set of IoT devices, i.e., $\mathscr{D}_{1 \leq i \leq n}$, which are the game players of $\mathbb{G}^{F}$.

(iii) $\mathbb{R}$ is the set of relays, i.e., $\mathscr{R}_{1 \leq i \leq n}$, and the second bargaining process is individually executed in each relay. In $\mathscr{R}_{i}$, the energy and data entities are game players of $\mathbb{G}_{i}^{S}$.

(iv) $\mathfrak{M}$ is the total bandwidth amount in the $S$ - $W$ joint platform where $\mathfrak{M}=\sum_{0 \leq i \leq n} \tau_{i}$.

(v) In $\mathbb{G}^{F}$, the strategy profile is $\left\{\tau_{1}, \ldots \tau_{i}, \ldots, \tau_{n}\right\}$ where $L_{t}^{\mathrm{UP}}=\sum_{1 \leq i \leq n} \tau_{i} . \tau_{i}$ is the size of the allocated time duration for the $\mathscr{D}_{i}$, and $U_{\mathscr{D}_{i}}$ is the utility function of $\mathscr{D}_{i}$.

(vi) In $\mathbb{G}_{i}^{S}$, the strategy is $\rho_{i}$ where $0 \leq \rho_{i} \leq 1$; it is the ratio for the $\mathscr{R}_{i}$ 's time splitting. $\mathfrak{U}_{\mathscr{R}_{i}}^{D}$ and $\mathfrak{U}_{\mathscr{R}_{i}}^{E}$ are utility functions of $\mathscr{R}_{i}{ }^{\prime} \mathrm{s}$ data and energy entities, respectively.

(vii) Discrete time model $T \in\left\{t_{1}, \ldots, t_{c}, t_{c+1}, \ldots\right\}$ is represented by a sequence of time steps. The length of a time step matches the $\mathbb{G}$ 's event time scale.

2.2. Basic Concepts of RABS and NABS. In this section, we introduce the two bargaining solution concepts employed in this study. $n$ game players, $i=1, \ldots, n$, need to agree on a payoff vector from a set of potential possibilities, $S \subseteq \mathbb{R}_{+}^{n}$. The 


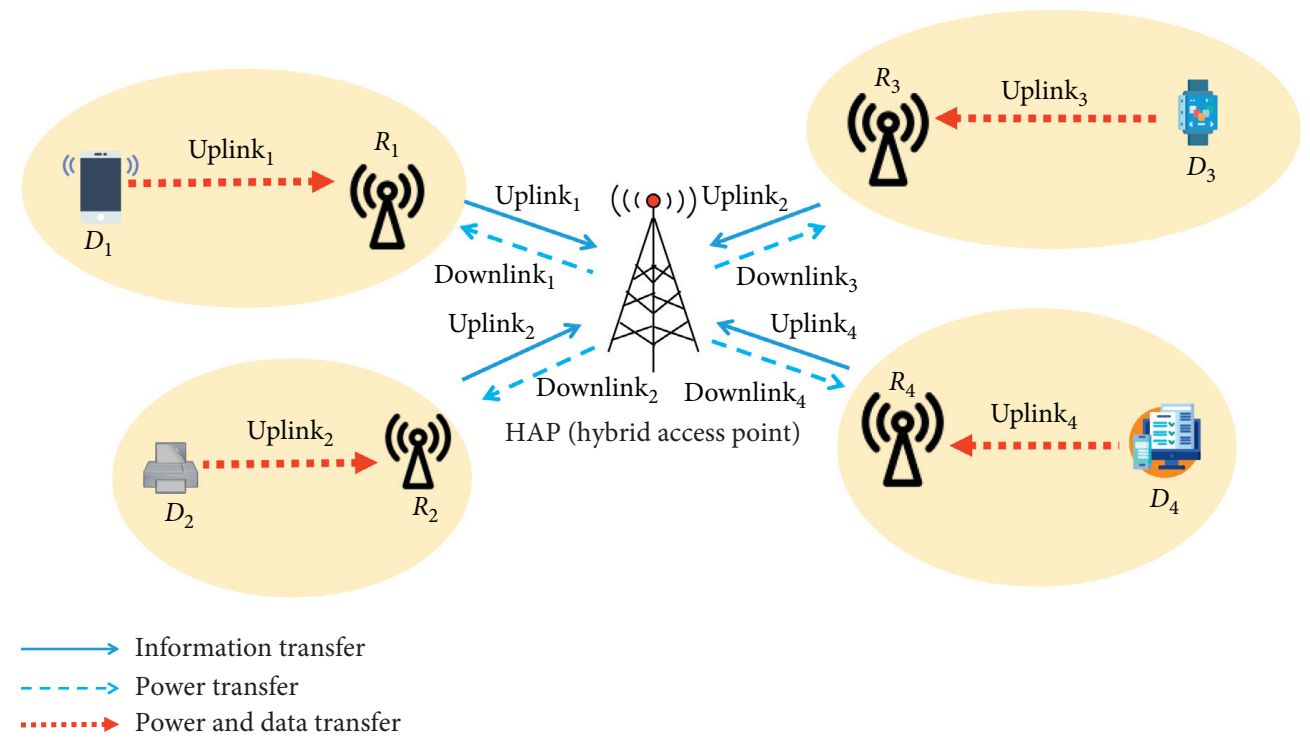

FIgURE 1: The dual-hop wireless communication platform.

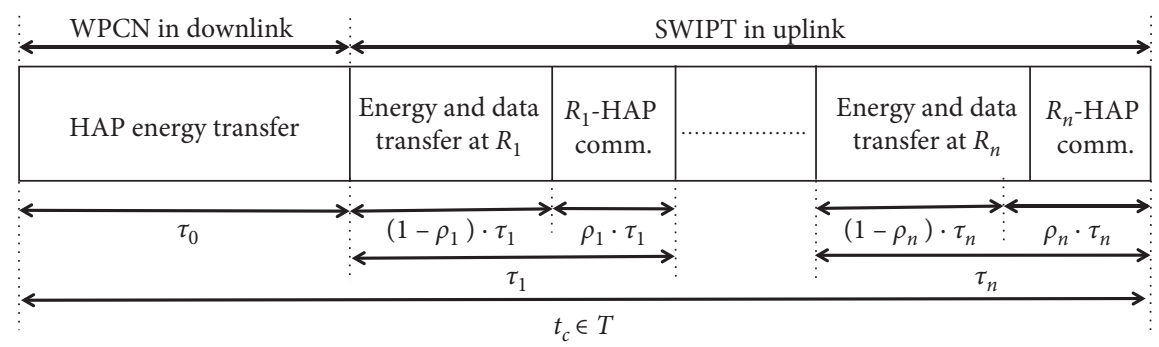

FIgURE 2: The downlink and uplink frame structure in the $S$ - $W$ joint platform.

disagreement point $d \in S$ is the outcome that the players get if they do not reach an agreement. Formally, a bargaining problem is a pair $(S, d)$ where the following two properties are fulfilled; (i) there exists $x \in S$ with $x \gg d$ where $y \gg x$ if $y_{i}>x_{i}$, and (ii) for every $x \in S$ and every $y \in \mathbb{R}^{n}$ with $d \leq y \leq x$, it follows that $y \in S$. Let $\Sigma^{n}$ be the family of all $n$-person bargaining problems of the form $(S, d)$. A bargaining solution is a function $f: \Sigma^{n} \longmapsto \mathbb{R}_{+}^{n}$ satisfying $f(S, d) \in S$ for every $(S, d) \in \Sigma^{n}$; that is, given a bargaining problem $(S, d)$, the solution $f$ prescribes $f(S, d)[10,11]$.

For a given $S \in \Sigma^{n}$, the utopia point $m(S) \in \mathbb{R}_{+}^{n}$ is the point where each coordinate $i$ contains the maximum conceivable outcome for the player $i$ among individually rational alternatives. Usually, the bargaining solution is strongly dependent on the bargaining powers. If different bargaining powers are used, the player with a higher bargaining power obtains a higher resource than the other players. To measure asymmetries in bargaining power, let $\alpha=\left(\alpha_{1}, \ldots, \alpha_{n}\right)$ be a vector of bargaining weights. The endogenous reference point $\left(m^{\alpha}(S)\right)$ is always feasible for every $S \in \Sigma_{0}$, i.e., $m^{\alpha}(S) \in S$. Formally, it is defined as follows [11]:

$$
\begin{aligned}
m^{\alpha}(S)= & \left(\left(\alpha_{1} \times m_{1}(S)\right), \ldots,\left(\alpha_{n} \times m_{n}(S)\right)\right), \\
& \text { s.t. }\left\{\alpha \in \Delta^{n-1}=\left\{\beta \in \mathbb{R}_{+}^{n} \mid \sum_{i=1}^{n} \beta_{i}=1\right\}, m_{i}(S)=\max \left\{x_{i} \mid x \geq d \text { and } x \in S\right\},\right\}
\end{aligned}
$$

where $m_{i}(S)$ is the player $i$ 's utopia point. At the endogenous reference point, each player achieves a fraction of his utopian payoff which is proportional to the players' bargaining power. In general, $m^{\alpha}(S)$ might be in the interior of $S$, and it captures a starting point for negotiated aspirations. For every $S \subset \mathbb{R}^{n}$, its Pareto optimal (PO) set is defined as $\mathbf{P O}(S)=\{y \in S \mid x$ $>y$ implies $x \notin S\}$, and its weakly Pareto optimal (WPO) set is defined as $\mathbf{W P O}(S)=\{y \in S \mid x \gg y$ implies $x \notin S\}$. A 
bargaining problem with a reference point is a triple $(S, d, r)$ where the reference point $r \in S \backslash \mathrm{WPO}(S)$ can satisfy $r \geq d$ $[10,11]$. The NABS identifies the PO point above $m^{\alpha}(S)$ which respects the given bargaining weights. With weights $\alpha \in \Delta^{n-1}$, the $\operatorname{NABS}(S, d, r)$ is mathematically given as follows [11]:

$$
\begin{aligned}
\operatorname{NABS}(S, d, r & \left.=m^{\alpha}(S)\right)=\left(\left(1-\lambda^{*}\right) \times m^{\alpha}(S)\right)+\left(\lambda^{*} \times m(S)\right), \\
\text { s.t. } \lambda^{*} & =\max \left\{\lambda \in[0,1] \mid\left[\left((1-\lambda) \times m^{\alpha}(S)\right)+(\lambda \times m(S))\right] \in S\right\} .
\end{aligned}
$$

Another bargaining solution, the RABS, also incorporates the reference point $(r)$ into the definition of bargaining problems. Particularly, $r$ is interpreted as an intermediate agreement which facilitates the conflict resolution process, and it has a strong potential to influence bargaining agreements. In addition, the RABS employs the aspiration point $\mathscr{A}(S, r)$, which is a new salient point into bargaining problems. For every $x \in S$, an aspiration vector $\mathscr{A}(S, r)$ is defined in such a way that for every $i \in\{1, \ldots, n\}: \mathscr{A}_{i}(S, x)=\max \left\{k \in \mathbb{R} \mid\left(k, x_{-i}\right) \in S\right\}$. Simply, the ideal point in the NABS is assumed as $\mathscr{A}(S, d)$. Since $r \geq d$, the aspiration point in the RABS will be lower compared to the ideal point in the NABS where $\mathscr{A}(S, r) \leq \mathscr{A}(S, d)$. With $\mathscr{A}(S, r)$, RABS $(S, d, r)$ is formally defined as follows [10]:

$$
\begin{aligned}
\operatorname{RABS}(S, d, r)= & \left(\Gamma^{*} \times \mathscr{A}(S, r)\right)+\left(\left(1-\Gamma^{*}\right) \times d\right), \\
\text { s.t. } \Gamma^{*}= & \max \{\Gamma \in[0,1] \mid[(\Gamma \times \mathscr{A}(S, r)) \\
& +((1-\Gamma) \times d)] \in S\} .
\end{aligned}
$$

Conceptually, for each game player, the ideal point, i.e., $\mathscr{A}(S, d)$, gives the maximum payoff level that can be reached in an individually rational agreement, and the aspiration point, i.e., $\mathscr{A}(S, r)$, is muted since $r \geq d$. Figure 3 provides a graphical illustration of the RABS and NABS for a twoplayer bargaining game.

2.3. Twofold Bargaining Game in the $S$-W Joint Platform. At each time period $t \in T$, IoT devices have their data information and attempt to transfer them to the HAP through their corresponding relays. In the frame structure, the first $\tau_{0}$ duration is dedicated for the WPCN downlink. During the time period $\tau_{0}$, the HAP broadcasts the RF energy signal, and the relays harvest the energy for their forwarding tasks. At time $t_{c}$, the $\mathscr{R}_{i}$ 's harvested energy function $\left(H_{\mathscr{R}_{i}}(\cdot)\right)$ from the HAP is expressed as follows:

$$
H_{\mathscr{R}_{i}}\left(\tau_{0}, \mathfrak{M}, d_{\mathscr{R}_{i}}, t_{c}\right)=\Delta_{e} \times \tau_{0} \times \mathfrak{M} \times\left(\frac{D_{M}^{\mathrm{HAP}}-d_{\mathscr{R}_{i}}}{D_{M}^{\mathrm{HAP}}}\right)^{\phi} \times t_{c},
$$

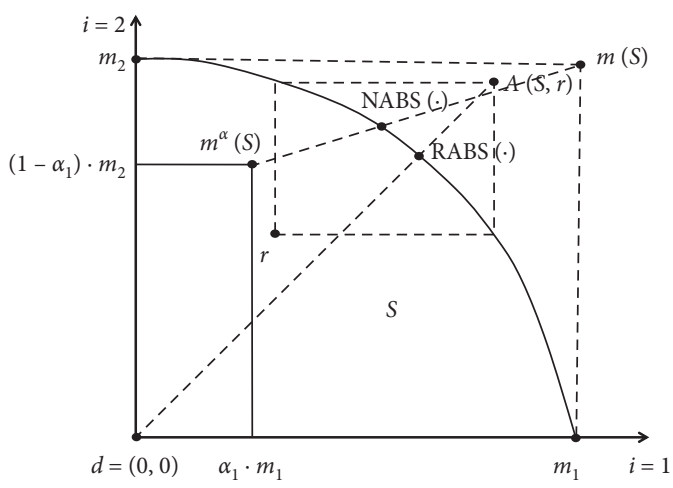

FIgURE 3: The RABS and NABS for a two-player bargaining game.

where $\Delta_{e}$ is the energy harvesting efficiency and $\phi$ is an adjustment factor for distance. $d_{\mathscr{R}_{i}}$ is the distance from the HAP to $\mathscr{R}_{i}$ and $D_{M}^{\mathrm{HAP}}$ is the maximum coverage range of HAP. The remaining time $\left(L_{t_{c}}^{\mathrm{UP}}\right)$ in the frame structure is assigned, (i) to transmit the data and energy from devices to relays and (ii) to retransmit the data from relays to the HAP. It is done by taking two time subslots for each device in the SWIPT uplink. First, $L_{t_{c}}^{\mathrm{UP}}$ is split into $n$ parts for each device; e.g., the time duration $\tau_{i}$ is dynamically allocated for the $\mathscr{D}_{i}$. Second, $\tau_{1 \leq i \leq n}$ is divided by the $\rho_{i}$ factor. These processes are formulated as $\mathbb{G}^{F}$ and $\mathbb{G}_{i}^{S}$ based on the twofold bargaining model. For $\mathbb{G}^{F}$, the utility function of $\mathscr{D}_{i}$ at time $t_{c}\left(U_{\mathscr{D}_{i}}(\cdot)\right)$ is formally defined as follows:

$$
\begin{aligned}
U_{\mathscr{D}_{i}}\left(\tau_{i}, t_{c}, \mathscr{Q}_{\mathscr{D}_{i}}^{t_{c}}, E_{\mathscr{R}_{i}}^{t_{c}}\right)= & \frac{\mathscr{X}}{\eta+\exp (\mathscr{X})} \\
& +\left(\left(\frac{E_{\mathscr{R}_{i}}^{T}-\mathrm{E}_{\mathscr{R}_{i}}^{t_{c}}}{E_{\mathscr{R}_{i}}^{T}}\right) \times \log (\mathscr{X}+\theta)\right), \\
\text { s.t. } \mathscr{X}= & \frac{\min \left(\left(\tau_{i} \times \mathfrak{M}\right), \mathscr{Q}_{\mathscr{D}_{i}}^{t_{c}}\right)}{\mathscr{Q}_{\mathscr{D}_{i}}^{t_{c}}},
\end{aligned}
$$

where $\mathscr{Q}_{\mathscr{D}_{i}}^{t_{c}}$ is the $\mathscr{D}_{i}$ 's data amount at time $t_{c}, \mathscr{E}_{\mathscr{R}_{i}}^{t_{c}}$ is the $\mathscr{R}_{i}$ 's remaining energy at time $t_{c}, E_{\mathscr{R}_{i}}^{T}$ is the maximum capacity of $\mathscr{R}_{i}$ 's battery, and $\eta$ and $\theta$ are control parameters for the $U_{\mathscr{D}_{i}}(\cdot)$. Usually, the utility function estimates the satisfaction of service quality. Therefore, it is defined as related to the amount of actually allocated time duration. To adaptively adjust the $\tau_{1 \leq i \leq n}$ values at each time period, we should consider the relative fairness among different IoT devices. Therefore, it is reasonable that the aspiration point is preferred to the utopian point to get the time splitting solution. Due to this reason, we adopt the idea of RABS for the first bargaining process. According to (3), the RABS solution for $\mathbb{G}^{F}$, i.e., $\operatorname{RABS}_{\mathbb{G}^{F}}(\cdot)$, is given by 


$$
\begin{aligned}
\operatorname{RABS}_{\mathbb{G}^{F}}\left(U_{\mathscr{D}_{1 \leq i \leq n}}(\cdot), S_{\mathbb{G}^{F}}, r_{\mathbb{G}^{F}}, d_{\mathbb{G}^{F}}\right) & =\max _{\Gamma \in[0,1]}\left\{\mathscr{D}_{i} \in \mathbb{N} \mid\left(\Gamma \times \mathscr{A}_{\mathscr{D}_{i}}\left(S_{\mathbb{G}^{F}}, r_{\mathbb{G}^{F}}\right)\right)+\left((1-\Gamma) \times d_{\mathbb{G}^{F}}\right)\right\}, \\
\text { s.t. } \mathscr{A}_{\mathscr{D}_{i}}\left(S_{\mathbb{G}^{F}}, r_{\mathbb{G}^{F}}\right) & =\max \left\{U_{\mathscr{D}_{i}}(\cdot) \in \mathbb{R} \mid\left(U_{\mathscr{D}_{i}}(\cdot),\left(r_{\mathbb{G}^{F}}\right)_{-i}\right) \in S_{\mathbb{G}^{F}}\right\},
\end{aligned}
$$

where $S_{\mathbb{G}^{F}}, r_{\mathbb{G}^{F}}$, and $d_{\mathbb{G}^{F}}$ are the feasible payoff sets, reference, and disagree points in $\mathbb{G}^{F}$, respectively. In our game model,
$d_{\mathbb{G}^{F}}$ is set to zeros, and $r_{\mathbb{G}^{F}}$ is defined based on the relative traffic weight of each device. It is given by

$$
\begin{aligned}
& r_{\mathbb{G}^{F}}=\left\{r_{\mathbb{G}^{F}}^{\mathscr{D}_{1<i n}} \mid r_{\mathbb{G}^{F}}^{\mathscr{D}_{i}}=Y \times\left(\frac{Q_{\mathscr{D}_{i}}^{t_{c}}}{\eta+\exp \left(\mathscr{Q}_{\mathscr{D}_{i}}^{t_{c}}\right)}+\left(\left(\frac{E_{\mathscr{R}_{i}}^{T}-E_{\mathscr{R}_{i}}^{t_{c}}}{E_{\mathscr{R}_{i}}^{T}}\right) \times \log \left(Q_{\mathscr{D}_{i}}^{t_{c}}+\theta\right)\right)\right)\right\}, \\
& \text { s.t. } Y=\frac{\mathscr{Q}_{\mathscr{D}_{i}}^{t_{c}}}{\sum_{\mathscr{D}_{j} \in \mathbb{N} \mathbb{Q}_{\mathscr{D}_{j}}^{t_{c}}}} .
\end{aligned}
$$

From $\mathbb{G}^{F}$, IoT devices obtain their time durations $\left(\tau_{1 \leq i \leq n}\right)$. And then, for the next round, the $n$ individual second bargaining processes are operated simultaneously and independently. At $\mathbb{G}_{1 \leq i \leq n}^{S}$, each individual relay $\mathscr{R}_{1 \leq i \leq n}$ decides its $\rho_{i}$ value to transfer the data and energy. The utility functions of data and energy entities in the $\mathscr{R}_{i}$, i.e., $\mathfrak{U}_{\mathscr{R}_{i}}^{D}(\cdot)$ and $\mathfrak{U}_{\mathscr{R}_{i}}^{E}(\cdot)$, are mathematically given as follows:

$$
\begin{aligned}
& \left\{\begin{array}{l}
\mathfrak{U}_{\mathscr{R}_{i}}^{D}\left(\rho_{i}, \tau_{i}, t_{c},{Q_{\mathscr{D}}}_{\mathscr{D}_{i}}^{t_{c}}\right)=\frac{\mu \times \mathscr{Y}}{\sigma+\exp (\mathscr{Y})}+\left(\frac{\mathscr{Q}_{\mathscr{D}_{i}}^{t_{c}}}{\tau_{i} \times \mathfrak{M}} \times \log (\mathscr{Y}+\xi)\right) . \\
\mathfrak{U}_{\mathscr{R}_{i}}^{E}\left(\rho_{i}, \tau_{i}, t_{c}, \mathrm{E}_{\mathscr{R}_{i}}^{t_{c}}\right)=\frac{\exp (\mathscr{Z})-\exp (-\mathscr{Z})}{\exp (\mathscr{Z})+\exp (-\mathscr{Z})}-\left(\left(\frac{d_{\mathscr{D}_{i}}}{D_{M}^{\mathscr{D}_{i}}}\right)^{\phi} \times \log (\mathscr{Z}+\beta)\right),
\end{array}\right. \\
& \text { s.t. }\left\{\begin{array}{l}
Y=\frac{\min \left(\left(\rho_{i} \times \tau_{i} \times \mathfrak{M}\right), Q_{\mathscr{D}_{i}}^{t_{c}}\right)}{Q_{\mathscr{D}_{i}}^{t_{c}}}, \\
Z=\frac{\min \left(E_{\mathscr{R}_{i}}^{T},\left(\mathscr{H}_{\mathscr{R}_{i}}^{\mathscr{D}_{i}}\left(\rho_{i}, \tau_{i}, d_{\mathscr{D}_{i}}, t_{c}\right)+\mathrm{E}_{\mathscr{R}_{i}}^{t_{c}}\right)\right)}{E_{\mathscr{R}_{i}}^{T}},
\end{array}\right.
\end{aligned}
$$

where $\mu, \sigma$, and $\xi$ are control parameters for $\mathfrak{U}_{\mathscr{R}_{i}}^{D}(\cdot)$ and $\phi$ and $\beta$ are adjustment factors for $\mathfrak{U}_{\mathscr{R}_{i}}^{E}(\cdot) \cdot d_{\mathscr{D}_{i}}$ is the distance from $\mathscr{D}_{i}$ to $\mathscr{R}_{i}$, and $D_{M}^{\mathscr{D}_{i}}$ is the maximum coverage range of $\mathscr{D}_{i} \cdot \mathscr{H}_{\mathscr{R}_{i}}^{\mathscr{D}_{i}}(\cdot)$ is the $\mathscr{R}_{i}$ 's energy harvesting function from the $\mathscr{D}_{i}$. From the viewpoint of each relay, its energy can be harvested according to the HAP and its corresponding device. Therefore, the $\mathscr{R}_{i}$ 's harvesting energy depends on the $\mathscr{H}_{\mathscr{R}_{i}}^{\mathscr{D}_{i}}(\cdot)$ and $H_{\mathscr{R}_{i}}$. At time $t_{c}, \mathscr{H}_{\mathscr{R}_{i}}^{\mathscr{D}_{i}}(\cdot)$ and the energy consumption function $\left(E_{\mathscr{R}_{i}}(\cdot)\right)$ for wireless communications between the HAP and $\mathscr{R}_{i}$ are given by

$$
\left\{\begin{array}{l}
\mathscr{H}_{\mathscr{R}_{i}}^{\mathscr{D}_{i}}\left(\rho_{i}, \tau_{i}, d_{\mathscr{D}_{i}}, t_{c}\right)=\Delta_{e} \times\left(1-\rho_{i}\right) \times \tau_{i} \times M \times\left(\frac{D_{M}^{\mathscr{D}_{i}}-d_{\mathscr{D}_{i}}}{D_{M}^{\mathscr{D}_{i}}}\right)^{\phi} \times t_{c}, \\
E_{\mathscr{R}_{i}}\left(\mathscr{Q}_{\mathscr{R}_{i}}, d_{\mathscr{R}_{i}}, t_{c}\right)=\Delta_{c} \times{Q_{\mathscr{D}_{i}}^{t_{c}}}^{t_{i}} \times\left(\frac{d_{\mathscr{R}_{i}}}{D_{M}^{\mathrm{HAP}}}\right)^{\phi},
\end{array}\right.
$$

where $\Delta_{c}$ is the energy consuming parameter for each data bit, and $Q_{\mathscr{D}_{i}}^{t_{c}}$, which is the $\mathscr{R}_{i}$ 's data amount at time $t_{c}$, is transferred to the HAP. In $\mathbb{G}_{i}^{S}$, it is worthless if the $\mathscr{R}_{i}$ 's 
energy is exhausted. Therefore, it is appropriate that disagree point should be decided according to the energy preference. Because of this, we use the concept of NABS to adjust the $\rho_{i}$ value. According to (2), the NABS solution for the $\mathbb{G}_{i}^{S}$, i.e., $\mathrm{NABS}_{\mathbb{G}_{i}^{S}}(\cdot)$, is given by

$$
\begin{aligned}
& \operatorname{NABS}_{\mathbb{G}_{i}^{s}}\left(\mathcal{U}_{\mathscr{R}_{i}}^{D}(\cdot), \mathfrak{U}_{\mathscr{R}_{i}}^{E}(\cdot), \rho_{i}, S_{\mathscr{R}_{i}}, r_{\mathscr{R}_{i}}, \mathscr{I}_{\mathscr{R}_{i}}, d_{\mathscr{R}_{i}}\right)=\max _{\rho_{i} \in[0,1]}\left\{\mathscr{R}_{i} \in \mathbb{R} \mid\left(\left(1-\rho_{i}\right) \times r_{\mathscr{R}_{i}}\right)+\left(\rho_{i} \times \mathscr{I}_{\mathscr{R}_{i}}\right)\right\}, \\
& \text { s.t. }\left\{\begin{array}{l}
m_{E}\left(S_{\mathscr{R}_{i}}\right)=\max \left\{\mathfrak{U}_{\mathscr{R}_{i}}^{E}(\cdot) \in \mathbb{R} \mid x \geq d_{\mathscr{R}_{i}} \text { and } x \in S_{\mathscr{R}_{i}}\right\} \\
m_{D}\left(S_{\mathscr{R}_{i}}\right)=\max \left\{\mathfrak{U}_{\mathscr{R}_{i}}^{D}(\cdot) \in \mathbb{R} \mid x \geq d_{\mathscr{R}_{i}} \text { and } x \in S_{\mathscr{R}_{i}}\right\} \\
\omega_{E}=\frac{E_{\mathscr{R}_{i}}^{T}-E_{\mathscr{R}_{i}}^{t_{c}}}{E_{\mathscr{R}_{i}}^{T}} \text { and } \omega_{D}=1-\omega_{E}, \\
r_{\mathscr{R}_{i}}=\left(\left(\frac{\omega_{E}}{\alpha} \times m_{E}\left(S_{\mathscr{R}_{i}}\right)\right),\left(\frac{\omega_{D}}{\alpha} \times m_{D}\left(S_{\mathscr{R}_{i}}\right)\right)\right), \\
\mathscr{I}_{\mathscr{R}_{i}}=\left(m_{E}\left(S_{\mathscr{R}_{i}}\right), m_{D}\left(S_{\mathscr{R}_{i}}\right)\right)
\end{array}\right.
\end{aligned}
$$

where $d_{\mathscr{R}_{i}}, \mathscr{I}_{\mathscr{R}_{i}}$, and $r_{\mathscr{R}_{i}}$ are the disagree, utopian, and reference points in the $\mathbb{G}_{i} S$, respectively, $\omega_{E}\left(\right.$ or $\left.\omega_{D}\right)$ is a weight control parameter for the energy (or data) entity, and $\alpha$ is a ratio correction factor for the $r_{\mathscr{R}_{i}} ; \omega_{E}$ and $\omega_{D}$ are used to decide the $r_{\mathscr{R}_{i}}$. According to (6) and (10), the RABS and NABS are obtained, and we use them to share the frame structure at time $t_{c}$ for the dual-hop communication. By iteratively repeating our twofold bargaining model, we can adapt the current $S$ - $W$ joint platform conditions while maximizing the system throughput.

\subsection{Main Steps of the Proposed S-W Joint Energy Transfer} Model. With different locations of IoT devices, the reliable and effective wireless communication is of great current and future interest. Since multiple relays are employed, IoT devices are deployed flexibly to meet the requirement of wireless converge for devices. In this paper, we exploit the $S$ $W$ joint platform to enhance dual-hop wireless communications. Particularly, we focus on the energy harvesting and data transmission in the uplink and present a novel twofold bargaining model to effectively handle the doubly near-far problem. At the first bargaining process, the RABS is used to adjust the time duration for each IoT device. At the second bargaining process, the NABS is adopted to decide the ratio of energy harvesting and data communication in each individual relay. According to our game-based approach, we can achieve a mutually desirable solution while flexibly sensing the dynamic changing system conditions. The main steps of our proposed scheme can be described as follows :

Step 1: for our simulation analysis, the values of adjustment parameters and control factors can be found in Table 1, and the simulation scenario is given in Section 3.
Step 2: the individual IoT devices are randomly located in the local area and generate independently their data information with different types and characteristics.

Step 3: at the time period $t_{c}$, the first $\tau_{0}$ duration is dedicated for the WPCN downlink. During $\tau_{0}$, the HAP broadcasts the RF energy signal and relays harvest their energy according to (4).

Step 4: the remaining time duration $\left(L_{t_{c}}^{\mathrm{UP}}\right)$ is designated for the SWIPT uplink. Based on the twofold game model, $L_{t_{c}}^{\mathrm{UP}}$ is shared to transmit data and energy.

Step 5: at the $\mathbb{G}^{F}$ process, the utility function of each $\mathscr{D}$ is defined by using (5), and the RABS is obtained to adjust the $\tau_{1 \leq i \leq n}$ values according to (6) and (7).

Step 6: at the $\mathbb{G}_{1 \leq i \leq n}^{S}$ process, the utility functions of data and energy entities in each $\mathscr{R}_{i}$ are defined by using $(8)$, and the NABS is gotten to decide the data and energy ratio $\left(\rho_{i}\right)$ according to (10).

Step 7: each $\mathscr{R}$ 's energy harvesting function from the corresponding device, and the energy consumption function are given by (9).

Step 8: during the twofold bargaining approach, the different network agents, i.e., $\mathscr{D}$ and $\mathscr{R}$, work together and act cooperatively with each other toward an appropriate dual-hop communication system performance.

Step 9: constantly, $\mathscr{D}$ and $\mathscr{R}$ are self-monitoring the current $S$ - $W$ joint platform environments, and proceed to Step 2 for the next twofold bargaining game process.

\section{Performance Evaluation}

In this section, we build a simulation model and evaluate the performance of our proposed scheme. To confirm the superiority of our twofold bargaining approach, we provide the 
TABLE 1: System parameters used in the simulation experiments.

\begin{tabular}{lcc}
\hline Parameter & Value & Description \\
\hline$n$ & 5 & The total number of IoT devices and relays \\
$\tau_{0}$ & 0.2 seconds & Time duration for the WPCN downlink \\
$\Delta_{e}$ & 0.3 & The energy harvesting efficiency \\
$\Delta_{c}$ & 1 & The energy consuming parameter for each data bit \\
$\phi$ & 2 & Adjustment factor for distance \\
$D_{M}^{\mathrm{HAP}}, D_{M}^{\mathscr{D}}$ & $10 \mathrm{~m}, 10 \mathrm{~m}$ & The maximum coverage ranges of HAP and $\mathscr{D}$, respectively \\
$\eta, \theta$ & 1,1 & Control parameters for the $U_{\mathscr{R}}(\cdot)$ \\
$\mu, \sigma, \xi$ & $2,1,1$ & Control parameters for the $\mathfrak{U}_{\mathscr{R}}^{D}(\cdot)$ \\
$\beta$ & 1 & Adjustment factor for the $\mathfrak{U}_{\mathscr{R}}^{E}(\cdot)$ \\
$\alpha$ & 10 & A ratio correction factor for $r_{\mathscr{R}}$ \\
$\tau_{0}$ & 0.2 & The size of WPCN downlink at time $t$ \\
$E_{\mathscr{R}}^{T}$ & $1 \mathrm{mAh}$ & The maximum battery capacity of $\mathscr{R}$ \\
$\mathscr{B}_{T}$ & $10 \mathrm{msec}$ & The basic unit for the time allocation process \\
\hline Data type & 5 & Service duration $(\mathrm{t})$ \\
1 & 10 & 10 \\
2 & 30 & 40 \\
3 & 10 & 45 \\
4 & 15 & 60 \\
5 & 25 & 90 \\
6 & Bandwidth requirements $(\mathrm{Mbps})$ & 35 \\
\hline
\end{tabular}

simulation results by comparing the DSTM, RDTM, and WCTM protocols in [6-8]. The assumptions of our simulation environments are as follows:

(i) The simulated $S$ - $W$ joint platform consists of one HAP, five IoT devices, and five relays where $|\mathbb{N}|=5$ and $|\mathbb{R}|=5$.

(ii) IoT devices are randomly located over the local area that is ten square meters. They are connected to their corresponding relays, which are located in the intermediate point between their corresponding devices and the HAP.

(iii) At each time period $t, \tau_{0}$ for the WPCN downlink is fixed as one-fifth of $t$.

(iv) The maximum coverage range of HAP and $\mathscr{D}$, i.e., $D_{M}^{\mathrm{HAP}}$ and $D_{M}^{\mathscr{D}}$, is set to 10 meters.

(v) The total bandwidth amount in the HAP ( $\mathfrak{M})$ is $10 \mathrm{Gbps}$, and the maximum battery capacity of $\mathscr{R}$ $\left(E_{\mathscr{R}}^{T}\right)$ is $1 \mathrm{G}$ mAh. Initially, all batteries in relays are completely recharged.

(vi) Each IoT device $\mathscr{D}_{1 \leq n \leq n}$ generates its data for dualhop communications. The generation process for data services is Poisson with rate $\Lambda$ (services/t), and the range of offered services was varied from 0 to 3.0 .

(vii) The disagree point profile in the bargaining process is set to zeros.

(viii) Six different kinds of data services are assumed based on their bandwidth requirements and service duration times; they are assumed as the $S-W$ joint platform's traffic load.

(ix) Each time period $t$ is assumed as one second.

(x) To reduce computation complexity, the amount of time allocation is specified in terms of basic time unit $\left(\mathscr{B}_{T}\right)$, where one $\mathscr{B}_{T}$ is the minimum amount (e.g., 10 milliseconds in our system) of allocation process.

(xi) System performance measures obtained on the basis of 100 simulation runs are plotted as a function of the offered service request load.

(xii) We assume the absence of physical obstacles in the wireless communications.

In Figure 4, we investigate the effects of dual-hop communication protocols to maximize the system throughput. It is observed that our twofold bargaining approach obtains a better system performance under higher data rates. It means that the proposed scheme can effectively handle the doubly near-far problem in the heavy-load traffic situation while substantially increasing the throughput of dual-hop communication platform. The evaluation results of normalized IoT device payoff are shown in Figure 5. From low to heavy data load distributions, the simulation results indicate that our proposed scheme performs better than the existing DSTM, RDTM, and WCTM schemes.

In Figure 6, we depict the relative fairness among IoT devices. The major challenge to develop our proposed scheme is to provide the most proper combination of the system efficiency and device fairness. It is a desirable property to operate multiple IoT devices in the dual-hop communication system. The curves presented in Figure 6 clearly show that our proposed scheme, in general, appropriately maintains the fairness among different IoT devices than other existing state-of-the art dual-hop communication protocols.

From the simulation results in Figures 4-6, it is evident that we can effectively compromise conflicting objectives to provide the most proper fair-efficient control solution for the $S-W$ joint system. However, research on the energy harvesting problem in the dual-hop communication 


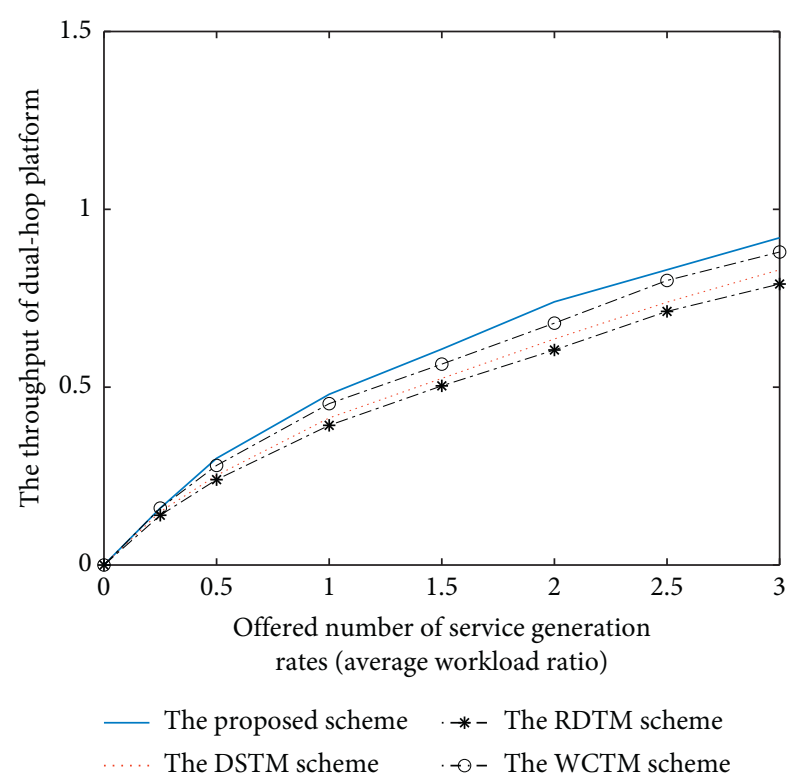

Figure 4: The throughput of dual-hop platform.

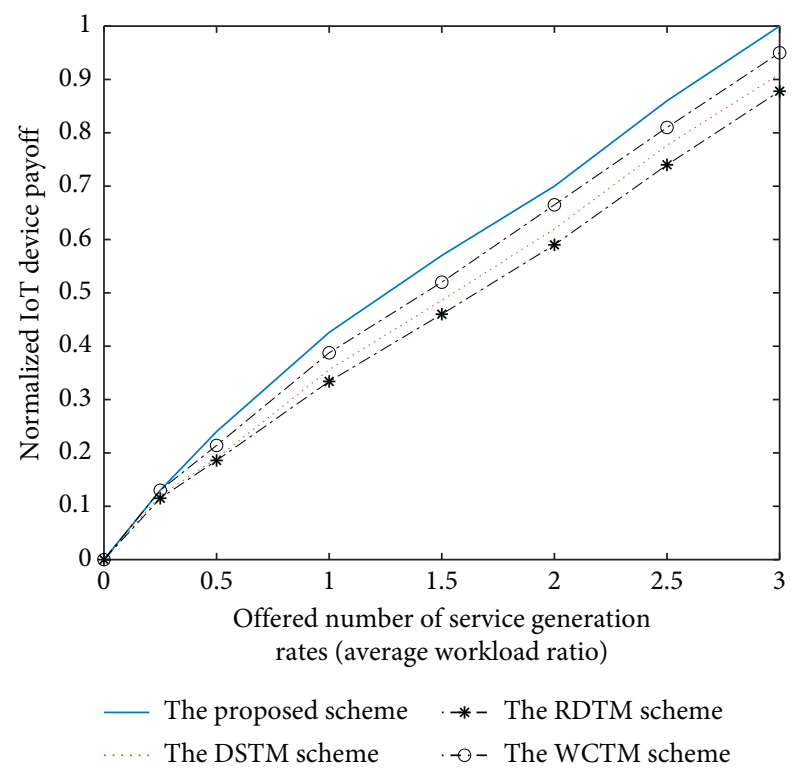

Figure 5: Normalized IoT device payoff.

platform is still in its infancy. An interesting continuation of our study presented in the paper can be extended in a number of ways. One future direction is to investigate the nonlinear energy harvesting models. Usually, linear energy harvesting models are too ideal in practical systems. There are some nonlinear energy harvesting models reported in $[16,17]$; they make it possible to transform the nonconvex joint optimization problem into a convex one. The piecewise linear energy harvesting model is one of the most analytically tractable models while ensuring a good accuracy.

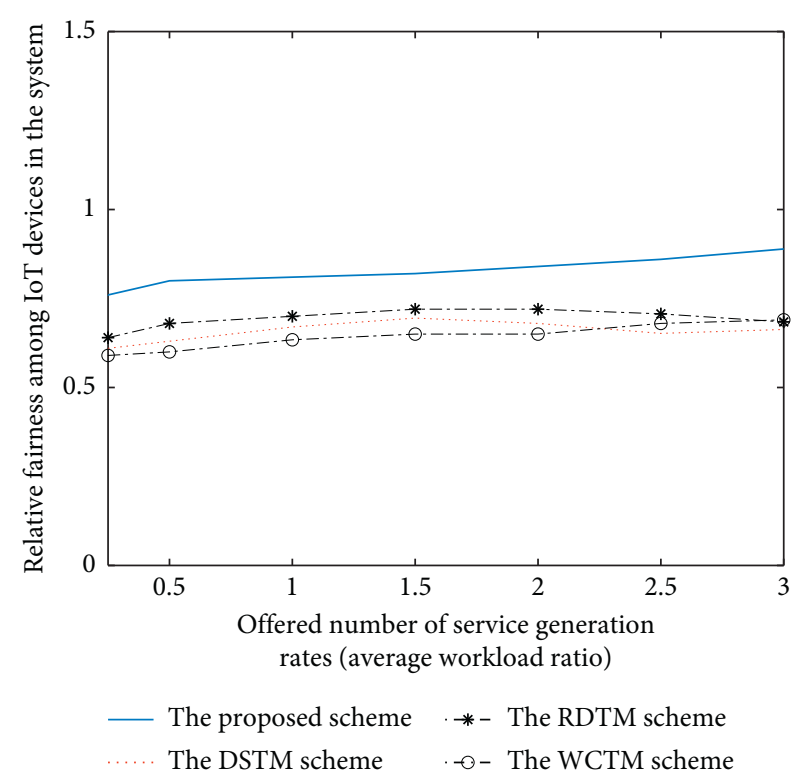

FIgURE 6: Relative fairness among IoT devices.

\section{Summary and Conclusions}

In this paper, we propose a novel twofold bargaining game model to share the uplink time in the S-W joint infrastructure. Based on the dual-hop communication platform, relays scavenge their required energy from the RF-enabled WPCN downlink, and the energy-carrying SWIPT uplink. In the latter case, the limited uplink is shared according to our twofold bargaining approach. At the first bargaining process, the basic idea of RABS is used to split the uplink capacity among multiple IoT devices. At the second bargaining process, the concept of NABS is adopted to share the allocated time duration to provide greater and reciprocal advantages. The main goal of our proposed scheme is to compromise a tradeoff between the energy harvesting and the data relaying; it is essential to operate the dual-hop communications while handling the doubly near-far problem. During the step-by-step interactive operation, our first and second bargaining processes are repeated sequentially to maximize the $S$ - $W$ joint system performance. Based on the simulation results presented in this article, it is concluded that our proposed scheme can get an excellent system performance than the existing DSTM, RDTM, and WCTM protocols under widely diversified data load intensities.

\section{Data Availability}

Data and material are available from the author at swkim01@sogang.ac.kr.

\section{Conflicts of Interest}

The author declares no conflicts of interest. 


\section{Acknowledgments}

This research was supported by the MSIT (Ministry of Science and ICT), Korea, under the ITRC (Information Technology Research Center) support program (IITP-20212018-0-01799) supervised by the IITP (Institute for Information and Communications Technology Planning and Evaluation).

\section{References}

[1] L. Chettri and R. Bera, "A comprehensive survey on Internet of Things (IoT) toward 5G wireless systems," IEEE Internet of Things Journal, vol. 7, no. 1, pp. 16-32, 2020.

[2] J. Yang, J. Hu, K. Lv, Q. Yu, and K. Yang, "Multi-dimensional resource allocation for uplink throughput maximisation in integrated data and energy communication networks," IEEE Access, vol. 6, pp. 47163-47180, 2018.

[3] S. Kumar, P. Tiwari, and M. Zymbler, "Internet of Things is a revolutionary approach for future technology enhancement: a review," Journal of Big Data, vol. 6, no. 111, pp. 1-21, 2019.

[4] H. Ju and R. Zhang, "Throughput maximization in wireless powered communication networks," IEEE Transactions on Wireless Communications, vol. 13, no. 1, pp. 418-428, 2014.

[5] Y. Ma, H. Chen, Z. Lin, Y. Li, and B. Vucetic, "Distributed and optimal resource allocation for power beacon-assisted wireless-powered communications," IEEE Transactions on Communications, vol. 63, no. 10, pp. 3569-3583, 2015.

[6] P. Ramezani and A. Jamalipour, "Throughput maximization in dual-hop wireless powered communication networks," IEEE Transactions on Vehicular Technology, vol. 66, no. 10, pp. 9304-9312, 2017.

[7] H. Yu, M. Ju, and C. Jeong, "Sum-throughput maximization in dual-hop wireless powered DF-based relay networks," IEEE Access, vol. 7, pp. 162268-162281, 2019.

[8] B. Lyu, T. Qi, H. Guo, and Z. Yang, "Throughput maximization in full-duplex dual-hop wireless powered communication networks," IEEE Access, vol. 7, pp. 158584-158593, 2019.

[9] S. Kim, Game Theory Applications in Network Design, IGI Global, Hershey, PA, USA, 2014.

[10] E. Karagozoglu and K. Keskin, "A tale of two bargaining solutions," Games, vol. 6, no. 2, pp. 161-174, 2015.

[11] C. Alós-Ferrer, J. García-Segarra, and M. Ginés-Vilar, “Anchoring on utopia: a generalization of the kalai-smorodinsky solution," Economic Theory Bulletin, vol. 6, no. 2, pp. 141-155, 2018.

[12] P. V. Balakrishnan, J. C. Gómez, and R. V. Vohra, "The tempered aspirations solution for bargaining problems with a reference point," Mathematical Social Sciences, vol. 62, no. 3, pp. 144-150, 2011.

[13] Z. Liu, S. Zhao, Y. Yuan, Y. Yang, and X. Guan, "Game-based approach of fair resource allocation in wireless powered cooperative cognitive radio networks," International Journal of Electronics and Communications, vol. 134, pp. 1-11, 2021.

[14] H. Chen, Y. Li, Y. Jiang, Y. Ma, and B. Vucetic, "Distributed power splitting for SWIPT in relay interference channels using game theory," IEEE Transactions on Wireless Communications, vol. 14, no. 1, pp. 410-420, 2015.

[15] Y. Xu, H. Xie, C. Liang, and F. Richard Yu, "Robust secure energy efficiency optimization in SWIPT-aided heterogeneous networks with a non-linear energy harvesting model," IEEE Internet of Things Journal, 2021.
[16] Y. Ye, L. Shi, X. Chu, and G. Lu, "Throughput fairness guarantee in wireless powered backscatter communications with HTT," IEEE Wireless Communications Letters, vol. 10, no. 3, pp. 449-453, 2021.

[17] H. Yang, Y. Ye, X. Chu, and M. Dong, "Resource and power allocation in SWIPT-enabled device-to-device communications based on a nonlinear energy harvesting model," IEEE Internet of Things Journal, vol. 7, no. 11, pp. 10813-10825, 2020. 